\title{
Clopidogrel associated leukopenia/neutropenia following coronary angioplasty
}

\section{Learning points for clinicians}

a. Clopidogrel induced bone marrow suppression is a very rare adverse effect. However, it should be considered in the differential diagnosis of a patient presenting with unexplained neutropenic sepsis who recently started Clopidogrel therapy.

b. Neutropenia can occur up to five weeks following the initiation of Clopidogrel.

c. Ticagrelor can be used as an alternative medication to Clopidogrel in patients experiencing adverse haematological reaction to Clopidogrel.

\section{Case report}

A 72 year old male was referred to the Emergency Department complaining of fever, shivering, generalised malaise, weakness, myalgia and dry cough ongoing for the previous three days. Five weeks prior to his presentation, the patient underwent coronary angiography which showed ninety percent stenosis of the mid-Left Anterior Descending Artery. He subsequently received angioplasty with a drug eluding stent. The patient was commenced on Clopidogrel $75 \mathrm{mg}$ PO once daily, in addition to his regular medications. He had no known drug allergies, he denied any other symptoms and his systemic review and physical examination was unremarkable. His full blood

Table I Blood count was normal prior to starting treatment with Clopidogrel
Volume 2 Issue I - 2018

\author{
Moneeb Mustafa, Inas Ragab, Megan Marshal, \\ Maged Esak, Martin Ryan \\ Department of Internal Medicine, St Luke General Hospital, \\ Ireland
}

Correspondence: Moneeb Mustafa, Department of Internal Medicine, St Luke General Hospital, Ireland;

Email muneeebseif@hotmail.com

Received: February 02, 2018 | Published: February 14, 2018

count on admission is shown in the table below. His kidney function, liver function, blood cultures and urine culture were normal. The patient was diagnosed with febrile neutropenia and was admitted to an isolation room. Clopidogrel was discontinued and he was commenced on Piperacillin/Tazobactam plus Gentamycin. The patient was supported with Filgrastrim and his blood count was monitored daily. The patient's blood count had recovered following six days of therapy and his fever subsided. Filgrastim and antibiotics were discontinued at that time. Following this, the patient maintained a stable blood count with no further drop or recurrence of fever. The patient was discharged on day ten with Ticagrelor in lieu of Clopidogrel and was monitored closely (Table 1).

\begin{tabular}{lllll}
\hline & WBC & Neutrophils & RBC & Platelets \\
\hline Prior to Clopidogrel initiation & $7.0 \times 10^{\wedge} 9 / \mathrm{L}$ & $4.81 \times 10^{\wedge} 9 / \mathrm{L}$ & $5.33 \times 10^{\wedge} 9 / \mathrm{L}$ & $275 \times 10^{\wedge} 9 / \mathrm{L}$ \\
On admission & $0.4 \times 10^{\wedge} 9 / \mathrm{L}$ & $0.01 \times 10^{\wedge} 9 / \mathrm{L}$ & $4.74 \times 10^{\wedge} 9 / \mathrm{L}$ & $187 \times 10^{\wedge} 9 / \mathrm{L}$ \\
Day 3 after stop Clopidogrel & $0.4 \times 10^{\wedge} 9 / \mathrm{L}$ & $0.02 \times 10^{\wedge} 9 / \mathrm{L}$ & $4.13 \times 10^{\wedge} 9 / \mathrm{L}$ & $178 \times 10^{\wedge} 9 / \mathrm{L}$ \\
ON Discharge (10 days) & $10.6 \times 10^{\wedge} 9 / \mathrm{L}$ & $7.90 \times 10^{\wedge} 9 / \mathrm{L}$ & $4.6 \times 10^{\wedge} 9 / \mathrm{L}$ & $346 \times 10^{\wedge} 9 / \mathrm{L}$ \\
One month after discharge & $10.1 \times 10^{\wedge} 9 / \mathrm{L}$ & $7.87 \times 10^{\wedge} 9 / \mathrm{L}$ & $4.36 \times 10^{\wedge} 9 / \mathrm{L}$ & $299 \times 10^{\wedge} 9 / \mathrm{L}$ \\
\hline
\end{tabular}

\section{Discussion}

Clopidogrel is a second generation thienopyridine that inhibits platelet aggregation. It is a mainstay of therapy in combination with Aspirin, in patients with Coronary Artery Disease, Acute Coronary Syndromes and/or after Percutaneous Coronary Intervention (PCI). Clopidogrel has many side effects, some of which are rare but severe including neutropenia that can occur between four weeks to three months after initiation of therapy. ${ }^{1}$ Clopidogrel was considered the most likely causative agent responsible for the severe haematological injury in our patient, as following discontinuation and three days of G-CSF therapy, neutrophil count rose and normalised within ten days. The count remained in the normal range following subsequent checks at one, two and four weeks. The incidence of Clopidogrel induced thrombocytopenia and neutropenia was reported to be $0.26 \%$ and $0.10 \%$, respectively in the CAPRIE study. ${ }^{2}$ The mechanism by which Clopidogrel induces bone marrow suppression is not well understood

but possibilities include cumulative toxicity or idiosyncratic reactions. ${ }^{3}$ Neutropenia can occur five weeks following initiation of Clopidogrel therapy. ${ }^{4}$

In the literature, there are few reported cases of haematologic abnormalities due to Clopidogrel, including rare cases of neutropenia $(0.1 \%)$, thrombocytopenia $(0.2 \%)$, one case of silent aplastic anaemia and two cases of febrile pancytopenia. ${ }^{3-8}$ In the PLATO trial ${ }^{9}$ and four phase II Ticagrelor trials, ${ }^{10}$ a very small percentage of patients experienced clinically important shifts in haematologic parameters (Haemoglobin, WBC and Platelet counts) and there were no treatment differences in these shifts throughout. In our patient, we used Ticagrelor 90mg PO twice daily as an alternative treatment to Clopidogrel, based on the evidence that haematological disorders, such as leukopenia/ neutropenia, anaemia, aplastic anaemia, agranulocytosis and thrombocytopenia are not listed as adverse reactions in the Ticagrelor prescribing information and a report from a previous case study ${ }^{8}$. 
Furthermore, based on our patient's follow-up, treatment has clearly been effective with no reduction in any haematological parameter.

\section{Summary of patient's blood count}

Blood count was normal prior to starting treatment with Clopidogrel. There is marked leucopenia/neutropenia after 5 weeks from treatment initiation. The following blood count results showed dramatic improvement after discontinuation of Clopidogrel with normalization of the figures prior to discharge. Follow up blood counts at 4 weeks showed stable blood count figures while patient was on treatment with Ticagrelor.

\section{Acknowledgements}

None.

\section{Conflict of interest}

The author declares no conflict of interest.

\section{References}

1. Sharis PJ, Cannon CP, Loscalzo J. The antiplatelet effects of ticlopidine and clopidogrel. Ann Intern Med. 1998;129(5):394-405.

2. CAPRIE Seering Committee. A randomised, blinded, trial of clopidogrel versus aspirin in patients at risk of ischaemic events (CAPRIE). Lancet. 1996;348(9038):1329-1339.
3. Zacharia G, Randhawa A, Marino N, et al. Severe bone marrow suppression associated with use ofclopidogrel. Journal of Case Reports and Studies. 2016;4(4):2348-9820.

4. Massimo Montalto, Italo Porto, Antonella Gallo, et al. Clopidogrel Induced Neutropenia after Coronary Stenting: Is Cilostazol a Good Alternative? International journal of vascular medicine. 2011;2011(2011):5.

5. McCarthy MW, Kockler DR. Clopidogrel associated Leukopenia. Ann Pharmacother. 2003;37(2):216-219.

6. Andres E, Perrin AE, Alt M, et al. Febrile pancytopenia associated with clopidogrel. Arch Intern Med. 2001;161(1):125.

7. Trivier JM, Caron J, Mahieu M, et al. Fatal aplastic anaemia associated with clopidogrel. Lancet. 2001;357(9254):446.

8. Shah R, Keough LA, Belalcazar Portacio A, et al. Ticagrelor as an alternative in clopidogrel associated neutropenia. Platelets. 2015;26(1):80-82.

9. Wallentin L, Becker RC, Budaj A, et al. Ticagrelor versus clopidogrel in patients with acute coronary syndromes. $N$ Engl $J$ Med. 2009;361(11):1045-1057.

10. Husted S, Emanuelsson H, Heptinstall S, et al. Pharmacodynamics, pharmacokinetics, and safety of the oral reversible P2Y 12 antagonist AZD6140 with aspirin in patient with atherosclerosis: a double-blind comparison to clopidogrel with aspirin. Eur Heart J. 2006;27(9):10381047. 